\title{
REVIEW
}

\section{Fascia Focused Manual Therapy Interventions- Proposed Treatment for Post-COVID Syndrome}

\author{
Sharkey John (iD \\ Faculty of Medicine, Dentistry and Clinical Sciences, University of Chester/NTC, Dublin, Ireland
}

\begin{abstract}
The novel virus identified as severe acute respiratory syndrome coronavirus [SARS-CoV-2] has resulted in the Coronavirus disease [COVID-19] worldwide pandemic. Confirmed cases of COVID-19 has surpassed 57 million people globally and numbers are exponentially increasing weekly. Significant numbers of recovering patients are reporting long-term, on-going painful soft tissue and respiratory complications. While the total number of deaths directly associated with COVID-19 has exceeded 1million people worldwide, a more accurate figure is estimated to be significantly higher due to limited testing and issues around accurately attributing cause of death. Since its earliest emergence experts have identified the primary source of entry to the body being the nasal and oral cavities via aerosol droplets proliferating across the respiratory tract. Similar Coronaviruses have typically caused mild enteric symptoms including nausea, vomiting, diarrhoea, and respiratory diseases including the common cold. In contrast, COVID-19 has been shown to cause acute fatal pneumonia, systemic out-of-control inflammatory responses, over-production of hyaluronan, cytokines, chemokines and C-reactive protein [CRP], leaky blood vessels, clot-causing antibodies leading to coagulation of blood, and resulting blood clots. Recorded high levels of serum Interleukin 6 [IL-6], Interleukin 8 [IL-8] as well as Tumour necrosis factor alpha [TNF- $\alpha$ ] and IL-1 $\beta$ have been observed in COVID-19 patients all contributing to connective tissue damage. The connective tissue fascia has been described as "the universal singularity" due to its ubiquitous nature and has been identified in virtually every organ and structure within the body. Based on available evidence this paper suggests that the Corona virus travels upon the highway that is the fascial singularity whence it permeates cells on a local and global level. A significant number of post-COVID-19 [SARS-CoV-2] patients will experience on-going fascia related pain and a wide range of functional issues. Based on current research this short report proposes that appropriate fascia focused manual and movement therapy interventions will assist patients in recovering from the COVID-19 [SARS-CoV-2]. Fascia focused therapeutic interventions are shown to reduce pain and support a reestablishment of function while providing safe, effective and appropriate non-invasive therapy.

A comprehensive search was conducted to systematically review research/reports and professional opinions related to postCOVID-19 syndrome. Surveying appropriate research and reports provided unique detail concerning fascial tissue involvement and underlying disease mechanisms including long-term after-effects of the novel Coronavirus disease [COVID-19].
\end{abstract}

KEYWORDS: Coronavirus. Fascia. Post-COVID Syndrome. Long-term COVID, post-COVID-19 syndrome. Hyaluronan. Angiotensin-converting enzyme 2 [ACE2]. Cytokines.

Correspondence: Dr John Sharkey, Faculty of Medicine, Dentistry and Clinical Sciences, University of Chester/NTC, 1516aSt Joseph’s Parade Dorset St DO7 FR6p Dublin, Ireland. Email: john.sharkey@ntc.ie

Copyright $@ 2021$ Sharkey J. This is an open access article distributed under the Creative Commons Attribution 4.0 International, which permits unrestricted use, distribution, and reproduction in any medium, provided the original work is properly cited.

\section{INTRODUCTION}

According to the European Centre for Disease Prevention and Control the number of confirmed cases of COVID-19 is in excess of 69.1 million people globally with 44.6 million in recovery. Of this number, in mid-December of 2020, 15.5 million accounted for the USA while the number of COVID-19 cases across Europe [in millions] was 69.1, UK 1.77, Brazil 6.73, Russia 2.52, France 2.32 and India was 9.77 reflecting the impact this pandemic continues to be inflicting on our local and global communities. A significant number of patients recovered from COVID-19 have reported long-term, on-going unresolved soft tissue and respiratory issues, a condition being called "long COVID" or "post-COVID syndrome" $[1,2]$. A sample group used to attain this data had a median age of 47 years with the majority having no previous health issues [2]. Debilitating symptoms such as sleep apnoea, disorientation, tachycardia, extreme fatigue, rash, blurred vision and impaired autoimmune issues have been shown to have a direct corelation with increased cytokines [3]. Of special interest to the manual and movement therapist unresolved symptoms include restricted painful motion, body-wide muscular and joint pain, impaired physical 
function and dyspnoea [2]. The exact molecular mechanisms for transporting SARS-CoV-2, via the eyes, mouth and nose, are under continual investigation [2]. However, the essential binding receptor allowing entry into the body has recently been identified as the protective protein angiotensin-converting enzyme 2 or ACE2 [4, 5]. Fascial tissue contains ACE2 mRNA within nasal and oral mucosa, nasopharynx, lungs, stomach, small intestine, colon, skin, lymphatics, blood vessels, thymus, bone, spleen, liver, kidney, and brain [4].

In addition, ACE2 is present in arterial and venous endothelial cells and arterial smooth muscle cells. ACE2 is abundantly present in the lung alveolar epithelial cells and small intestine epithelia as well as the cardiovascular system $[4,7]$.

This epithelial expression, together with the presence of ACE2 in vascular endothelium, provides early data helpful in understanding the pathogenesis of the main SARS disease manifestations such as inflammation [27]. ACE2 is also acknowledged for its role in controlling and maintaining blood pressure [6]. Based on previous research it is proposed that changes in fascia tissue densification, architectural changes, fibrotic formation and dehydration due, in part, to increased hyaluronan can result in local and global pain mechanisms [8]. Reduced tissue gliding coupled with faulty breathing mechanisms can induce pain along with changes in sensations as the result of dyspnoea [9]. It has been reported that $36.4 \%$ of recovering COVID-19 patients develop neurological symptoms that include headaches [10]. Due to the ubiquitous nature of fascia it has been described as the universal singularity and is now recognised as one of the body's most neurologically rich sources of sensory nerves $[28,29]$. Due to the complexity of post-COVID-19 issues it has been stated that post-COVID-19 patients will dominate medical practice for the foreseeable future [11]. Fascia-focused manual therapy interventions have been shown to be effective in the treatment of inflammatory mediated pain and non-specific pain mechanisms and, therefore, it is proposed, can provide effective and welcomed therapeutic interventions for alleviating and managing 'post-COVID syndrome' $[9,21,22,23,24,25$, $26]$. Incorporating qualified therapists, who are trained in the treatment of fascia, as part of the multidisciplinary team will provide much needed support in alleviating already over-stressed healthcare systems and their workers [31]. Additionally, the integration and use of telehealth patient-centred consultations will provide pre, post and "between session" therapy guidance, advise and recommendations valuable in supporting the goal of improved quality of life [13].

Failure in the ability of fascia to glide relative to deeper or more superficial tissue is now recognised as a significant contributor to myofascial pain and inflammation, as well as contributing to changes in sensations [12, 14]. Inflammation has been shown to promote the production of glycosaminoglycans, especially hyaluronan and bradykinins, within the extracellular matrix leading to leaky blood vessels, coagulation and possible clotting [15]. Histological investigations of cadaver lung tissue sections from patients who had COVID-19 supported previous findings of a significant increase in hyaluronan, as much as 20 times that of a healthy lung [15]. Hyaluronic acid or hyaluronan is naturally present in the skin, eyes and synovial joints [34]. A number of studies have confirmed the continuity of the fascia singularity $[17,18,19]$ and clearly demonstrate how pain in one region can be due to morphological, structural, tensional or chemical changes at a local or distant anatomical site [20]. Manual Therapists trained to specialise in the provision of fascia focused treatments are ideally placed to provide appropriate therapeutic interventions to help resolve resulting adhesive effects of respiratory and vascular secretions [16]. Studies support the efficacy of fascia focused manual interventions which not only improve gliding of tissues but also restore normal levels of hyaluronan $[32,33]$.

This review identified a connection between fascia and serum interleukins IL-6, IL-8, tumour necrosis factor $[\mathrm{TNF}]-\alpha$ and IL- $1 \beta$, hyaluronan and the pathophysiology of severe acute respiratory syndrome [SARS] [21, 22] and, importantly, an under-reported aspect of COVID-19, the development of inflammation of the superficial fascia due to an abnormal amassing of eosinophils [30]. Although there is little research on the topic a recent study showed a significant improvement of eosinophil levels using a combination of interdisciplinary pain management combined with physical therapies involving massage and appropriate physical activity [35].

A comprehensive assessment is the key to ensuring a 'person centred' approach to the provision of longCovid/post-COVID-19 fascia focused manual and movement therapeutic interventions. While all longCovid/post-COVID-19 patients share the experience of having contracted and survived COVID-19 each case must be considered on an individual basis [23]. While similarities abound, no two patients are ever the same [24] and no two treatments will be identical.

\section{CONCLUSION}

Following the arrival of the Coronavirus [COVID-19] worldwide pandemic buoyed by the inevitable and widely held opinion that "we are never going back to normality", the overwhelming evidence suggests that a new era of healthcare is rapidly dawning. Now is the time for effective steps to integrate qualified therapists with specific skills and knowledge related to myofascial therapies into the multidisciplinary team. A move away from the dominant musculoskeletal model towards a fascia focused model now not only seems inevitable but is of vital necessity. Failure to not include therapists appropriately trained in fascia focused therapy as an important member of the multidisciplinary team will increase stress on hospitals and the healthcare system as a whole. 


\section{ACKNOWLEDGMENTS}

None.

\section{AUTHORS' CONTRIBUTIONS}

The participation of the author corresponds to the criteria of authorship and contributorship emphasized in the Recommendations for the Conduct, Reporting, Editing, and Publication of Scholarly work in Medical Journals of the International Committee of Medical Journal Editors. Indeed, all the authors have actively participated in the redaction, the revision of the manuscript, and provided approval for this final revised version.

\section{COMPETING INTERESTS}

The author declares no competing interests with this case.

\section{FUNDING SOURCES}

None.

\section{REFERENCES}

[1] Carole H. Sudre, Benjamin Murray, Thomas Varsavsky, Mark S. Graham, Rose S. Penfold, Ruth C. Bowyer, Joan Capdevila Pujol, Kerstin Klaser, Michela Antonelli, Liane S. Canas, Erika Molteni, Marc Modat, M. Jorge Cardoso, Anna May, Sajaysurya Ganesh, Richard Davies, Long H Nguyen, David A. Drew, Christina M. Astley, Amit D. Joshi, Jordi Merino, Neli Tsereteli, Tove Fall, Maria F. Gomez, Emma L. Duncan, Cristina Menni, Frances M.K. Williams, Paul W. Franks, Andrew T. Chan, Jonathan Wolf, Sebastien Ourselin, Tim Spector, Claire J. Steves. Attributes and predictors of Long-COVID: analysis of COVID cases and their symptoms collected by the Covid Symptoms Study App medRxiv 2020.10.19.20214494;

[2] Yvonne M.J. Goërtz, Maarten Van Herck, Jeannet M. Delbressine, Anouk W. Vaes, Roy Meys, Felipe V.C. Machado, Sarah Houben-Wilke, Chris Burtin, Rein Posthuma, Frits M.E. Franssen, Nicole van Loon, Bita Hajian, Yvonne Spies, Herman Vijlbrief, Alex J. van 't Hul, Daisy J.A. Janssen, Martijn A. Spruit. Persistent symptoms 3 months after a SARS-CoV-2 infection: the post-COVID-19 syndrome? ERJ Open Research 20206 : 00542-2020;

[3] Krueger J. M. The role of cytokines in sleep regulation. 2008. Current pharmaceutical design, 14[32], 3408-3416.

[4] Hamming I, Timens W, Bulthuis ML, Lely AT, Navis G, van Goor H. Tissue distribution of ACE2 protein, the functional receptor for SARS coronavirus. A first step in understanding SARS pathogenesis. J Pathol. 2004 Jun;203[2]:631-7.

[5] Li, W., Moore, M., Vasilieva, N. et al. Angiotensinconverting enzyme 2 is a functional receptor for the SARS coronavirus. 2003. Nature 426, 450-454.

[6] Bosso M, Thanaraj TA, Abu-Farha M, Alanbaei M, Abubaker J, Al-Mulla F. The Two Faces of ACE2: The Role of ACE2 Receptor and Its Polymorphisms in Hypertension and COVID-19. Mol Ther Methods Clin Dev. 2020 Jun 25;18:321-327.

[7] Zhang, H., Li, H. B., Lyu, J. R., Lei, X. M., Li, W., Wu, G., Lyu, J., \& Dai, Z. M. [2020]. Specific ACE2 expression in small intestinal enterocytes may cause gastrointestinal symptoms and injury after 2019-nCoV infection. International journal of infectious diseases : IJID : official publication of the International Society for Infectious Diseases, 96, 19-24.

[8] Li LQ, Huang T, Wang YQ, Wang ZP, Liang Y, Huang TB, Zhang HY, Sun W, Wang Y. COVID-19 patients' clinical characteristics, discharge rate, and fatality rate of metaanalysis. J Med Virol. 2020 Jun;92[6]:577-583.

[9] Stecco C, Stern R, Porzionato A, Macchi V, Masiero S, Stecco A, De Caro R. Hyaluronan within fascia in the etiology of myofascial pain. Surg Radiol Anat. 2011 Dec;33[10]:891-6.

[10] Chaitow L, Bradley D, Gilbert C. Multidisciplinary Approaches to Breathing Pattern Disorders. London, UK: Churchill Livingstone; 2002 [Google Scholar]

[11] Sheehy LM. Considerations for Postacute Rehabilitation for Survivors of COVID-19. JMIR Public Health Surveill. 2020 May 8;6[2]:e19462

[12] Barker-Davies RM, O'Sullivan O, Senaratne KPP, Baker P, Cranley M, Dharm-Datta S, Ellis H, Goodall D, Gough M, Lewis S, Norman J, Papadopoulou T, Roscoe D, Sherwood D, Turner P, Walker T, Mistlin A, Phillip R,
Nicol AM, Bennett AN, Bahadur S. The Stanford Hall consensus statement for post-COVID-19 rehabilitation. $\mathrm{Br}$ J Sports Med. 2020 Aug;54[16]:949-959.

[13] Stecco C, Stern R, Porzionato Aet al. Hyaluronan within fascia in the etiology of myofascial pain. Surg Radiol Anat. 2011;33[10]:891-896

[14] Lew HL, Oh-Park M, Cifu DX. The War on COVID-19 Pandemic: Role of Rehabilitation Professionals and Hospitals. Am J Phys Med Rehabil. 2020 Jul;99[7]:571572.

[15] Guimberteau JC, Delage JP, Wong J. The role and mechanical behaviour of the connective tissue in tendon sliding. Chir Main. 2010;29[3]:155-166.

[16] Kaber G, Kratochvil MJ, Burgener EB, Peltan EL, Barlow G, Yang S, Nicolls MR, de Jesus Perez V, Rosser JI, Wardle AJ, Kalinowski A, Ozawa MG, Regula DP, Nagy N, Heilshorn SC, Milla CE, Rogers AJ, Bollyky PL. Hyaluronan is abundant in COVID-19 respiratory secretions. medRxiv [Preprint]. 2020 Sep 11:2020.09.

[17] Ajimsha MS, Daniel B, Chithra S. Effectiveness of myofascial release in the management of chronic low back pain in nursing professionals. J Bodyw Mov Ther. 2014 Apr; 18[2]:273-81.

[18] Stecco C, Porzionato A, Macchi V, Stecco A, Vigato E, Parenti A, Delmas V, Aldegheri R, De Caro R. The expansions of the pectoral girdle muscles onto the brachial fascia: morphological aspects and spatial disposition. Cells Tissues Organs. 2008; 188[3]:320-9.

[19] Stecco A, Gilliar W, Hill R, Fullerton B, Stecco C. The anatomical and functional relation between gluteus maximus and fascia lata. Jour Bodyw Mov Ther. 2013 Oct; 17[4]:512-7.

[20] Stecco C. Functional Atlas of the Human Fascial System. Edinburgh: Churchill Livingstone; 2015.

[21] Sharkey, J. Should bone be considered fascia: Proposal for a change in taxonomy of bone- a clinical anatomist's view. International Journal of Biological and Pharmaceutical Sciences Archive. 2020.

[22] Tozzi P. Selected fascial aspects of osteopathic practice. 2012. J Bodyw Mov Ther. 16[4]:503-519.

[23] Tozzi P, Bongiorno D, Vitturini C. Fascial release effects on patients with non-specific cervical or lumbar pain. 2011. J Bodyw Mov Ther;15[4]:405-416.

[24] Zügel, M., Maganaris, C. N., Wilke, J., Jurkat-Rott, K., Klingler, W., Wearing, S. C., Findley, T., Barbe, M. F., Steinacker, J. M., Vleeming, A., Bloch, W., Schleip, R., \& Hodges, P. W. Fascial tissue research in sports medicine: from molecules to tissue adaptation, injury and diagnostics: consensus statement. 2018. British journal of sports medicine, 52[23], 1497.

[25] Parravicini G, Bergna A. Biological effects of direct and indirect manipulation of the fascial system. Narrative review. J Bodyw Mov Ther. 2017 Apr;21[2]:435-445.

[26] Chaitow, L. New evidence of a dynamic fascial maintenance and self-repair process.J Bodyw Mov Ther. 2016 Oct;20[4]:701-703.

[27] Stecco, A., Stern, R., Fantoni, I., De Caro, R., Stecco, C. Fascial Disorders: Implications for Treatment. PM R. 2016 Feb;8[2]:161-8.

[28] Iwasaki, M., Saito, J., Zhao, H., Sakamoto, A., Hirota, K., \& Ma, D. Inflammation Triggered by SARS-CoV-2 and ACE2 Augment Drives Multiple Organ Failure of Severe 
COVID-19: 2020. Molecular Mechanisms and Implications. Inflammation, 1-22. Advance online publication.

[29] Mense S. Innervation of the thoracolumbar fascia. 2019. European journal of translational myology, 29(3), 8297.

[30] Yahia, L., Rhalmi, S., Newman, N., Isler, M. Sensory innervation of human thoracolumbar fascia: 1992. An immunohistochemical study. Acta Orthop Scand; 63:1957

[31] Fraissé, M., Logre, E., Mentec, H. et al. Eosinophilia in critically ill COVID-19 patients: a French monocenter retrospective study. 2020. Crit Care 24, 635.

[32] Talaee, N., Varahram, M., Jamaati, H., Salimi, A., Attarchi, M., Kazempour Dizaji, M., Sadr, M., Hassani, S., Farzanegan, B., Monjazebi, F., \& Seyedmehdi, S. M. (2020). Stress and burnout in health care workers during
COVID-19 pandemic: validation of a questionnaire. Zeitschrift fur Gesundheitswissenschaften = Journal of public health, 1-6.

[33] Roman, M., et al., 2013. Mathematical analysis of the flow of hyaluronic acid around fascia during manual therapy motions. J. Am. Osteopath. Assoc. 113 (8), 600e610.

[34] Chaudhry, H., et al., 2013. Squeeze film lubrication for non-Newtonian fluids with application to manual medicine. Biorheology 50 (3e4), 191e202.

[35] Gupta, R. C., Lall, R., Srivastava, A., \& Sinha, A. (2019). Hyaluronic Acid: Molecular Mechanisms and Therapeutic Trajectory. Frontiers in veterinary science, 6, 192.

[36] O'Laughlin, T.J., Klima, R.R., Kenney, D.E. Rehabilitation of eosinophilic fasciitis. A case report. Am J Phys Med Rehabil. 1994 Jul-Aug;73(4):286-92. 